\title{
On free groups of motions and decompositions of the Euclidean space
}

\section{by \\ Jan Mycielski and S. Świerczkowski (Wrocław)}

The purpose, of this paper is to prove two theorems given in section $1\left({ }^{1}\right)$. These theorems are solutions of some problems proposed to the anthors by T. Dekker.

1. A group $\Phi$ of 1-1 transformations of a set $E$ onto itself is called without fixed points if for every $p \in E$ and every $\varphi \in \Phi \backslash(e)$ we have $\varphi(p) \neq p$.

The rank of a free group is the potency of a set of free generators of this group.

The sense-preserving isometries of the Euclidean space $\mathscr{E}^{3}, i . e$. , the superpositions of rotations and translations are called motions.

THEOREM 1. There exists a free group of the ranle $2^{\aleph_{0}}$ of motions of $\mathcal{E}^{3}$ without fixed points.

The proof of this theorem follows in sections $3-9$ (it is an effective construction, which does not use the axiom of choice). The relations of Theorem 1 with known results are given in section 2 .

An application of this theorem (the construction of a set $E \subset \mathcal{G}^{\mathbf{3}}$ which is congruent to $(E, A) \cup B$ for any at most denumerable sets $\left.A, B \subset \mathcal{G}^{3}\right)$ is given in [7]. Another application, of a well known character (compare [2], [3], [4], [8]), is the theorem 2 of this paper. It states that, for any system of congruence relations, $\mathcal{E}^{3}$ cah be divided into disjoint sets satisfying that system. For an exact description of this theorem we take the following notations:

$M$ and $N$ are non-empty sets.

$\left\{P_{\mu}\right\}_{\mu \in M}$ and $\left\{Q_{\mu}\right\}_{\mu \in M}$ are arbitrary systems of subsets of $N$, all different from $\varnothing$ and $N$. (We do not suppose that $\mu_{1} \neq \mu_{2}$ implies $P_{\mu_{1}} \neq P_{\mu_{2}}$ or $Q_{\mu_{1}} \neq Q_{\mu_{2}}$.)

$\simeq$ denotes congruence of point sets realizable by a motion.

(1) They were announced in [6] by the first author, but the original proof was faulty, and the proof presented here was worked out by both authors. 
THeOREM 2. If $\bar{M} \leqslant 2^{\mathbf{x}_{0}}$ and $\bar{N} \leqslant 2^{\mathrm{K}_{0}}$ then the space $\mathcal{E}^{3}$ can be decomposed into $\overrightarrow{\bar{N}}$ disjoint sets $A_{\nu}(\nu \in N)$ satisfying the system of congruences

$$
\bigcup_{v \in P_{\mu}} A_{\nu} \simeq \bigcup_{v \in Q_{\mu}} A_{n} \quad(\mu \in M) .
$$

Moreover all the pieces $A_{y}$ can be non-empty.

The proof of this theorem follows in section 10. The relations of Theorem 2 with known results are given in section 2. Note here the following applications:

(A) $\mathcal{E}^{3}$ is the sum of a sequence $A_{1}, A_{2}, \ldots$ of disjoint sets such that

$$
\bigcup_{n \in N_{\mathbf{1}}} A_{n} \simeq \bigcup_{n \in N_{2}} A_{n}
$$

for any sets $N_{1}, N_{2} \subset\{1,2, \ldots\}$ different from $\emptyset$ and $\{1,2, \ldots\}$.

(B) For any order type $\alpha$ of potency $\leqslant 2^{\mathrm{X}_{0}}$ without the upper end, $\mathcal{E}^{3}$ is the sum of a family of distinct sets, ordered by the relation $C$ isomorpbically to $a$ and congruent by motions each to the other.

Indeed let $N$ be ordered in the type $\alpha$ by the relation $\rightarrow$ and $v_{0} \in N$ and $M=N$. Take for (1) the system

$$
\bigcup_{v \in Y_{,},\{\mu} A_{r} \simeq A_{v_{0}} \quad(\mu \in N) \text {. }
$$

(C) There exists such a set $E C \mathcal{E}^{3}$ that for any cardinal $\mathrm{m}$, such that $2 \leqslant m \leqslant 2^{N_{0}}, \mathcal{E}^{3}$ is a sum of $m$ disjoint sets each congruent by a motion with $E$.

Indeed let $\bar{N}=2^{N_{0}}, N_{\mathfrak{E}} \subset N, \ddot{N}_{\mathrm{f}}=£$ for any $<2^{N_{0}}$ and $v_{0} \in N$. Take for (1) the system

$$
\begin{gathered}
A_{v_{0}} \simeq A_{p} \quad(\nu \in N), \\
A_{v_{0}} \simeq \bigcup_{p \in N \backslash N_{\mathfrak{f}}} A_{p} \quad\left(\mathfrak{f}<2^{N_{0}}\right) .
\end{gathered}
$$

Then we put $E=A_{v_{0}}$ and (C) is obvious.

At last note that the generalization of Theorems 1 and 2 to any space $\mathcal{E}^{n}$ with $n \geqslant 3$ follows immediately.

2. Concerning Theorem 1 note that the existence of a free group of the rank $2^{\mathrm{X}_{0}}$ of rotations of $\mathscr{E}^{3}$ around a fixed point is well known (Sierpiński [12], p. 238 Lemme 1). Sierpiński's proof was simplified and related results were obtained by $J$. de Groot [5]. This theorem easily follows from our Theorem 1 (by the method given in section 5). These proofs are effective, $i . e$. , they do not use the axiom of choice. Noneffective theorems on the existence of free subgroups in topological groups, generalizing Sierpiński's theorem are given in [1].
As for Theorem 2, it is analogous to theorems known for spheres and non-Euclidean spaces of dimension $\geqslant 2$ ([2], [3]). It permits us to complete the two tables given in [3], p. 107 in all points concerning the Enclidean spaces. Theorem 2 follows from our Theorem 1 and a theorem of $\mathrm{T}$. Dekker ([2], 2.2.2), but we give a direct proof in section 10 because in our case it is simpler. The applications (A)-(C) are analogons to the statements about the sphere $\mathcal{S}_{2}$ (instead of $\mathscr{G}^{3}$ ) proved in [4] and [8]. Of course Theorem 2 for the space $\mathcal{E}^{3}$ without one point follows from the analogous result concerning the sphere $\mathcal{S}_{2}$, but then motions are not sufficient to realize all the congruences - in general reflections are needed.

Theorem 1 does not hold for $\mathcal{E}^{1}$ and $\mathscr{E}^{2}$ because the group of motions of the plane is solvable $([9]$, p. 10) and thus cannot contain any free group of rank $>1$. Neither Theorem 2 holds for the line and the plane, as has been proved by T. Dekker ([2], p. $\breve{584}$ ).

3. We put

$$
\begin{aligned}
& A_{\psi}=\left(\begin{array}{ccc}
\cos \varphi & -\sin \varphi & 0 \\
\sin \varphi & \cos \varphi & 0 \\
0 & 0 & 1
\end{array}\right), \quad B_{q}=\left(\begin{array}{ccc}
1 & 0 & 0 \\
0 & \cos \varphi & -\sin \varphi \\
0 & \sin \varphi & \cos \varphi
\end{array}\right), \\
& R_{q q}=A_{q} B_{w} A_{q}^{-1}, \\
& f(x)=\sum_{n=1}^{\infty} 2^{-[n x]_{1} 3^{n^{2}}} \quad \text { fo } \cdot \quad x>0 \\
& \varphi(x)=2 \operatorname{arctg} f(x) .
\end{aligned}
$$

Let $T$ be a translation of $\mathcal{E}^{3}$ obtained by adding to every point of $\mathcal{E}^{3}$ the point-vector $A_{q}(1,0,0)=$ the point $(1,0,0)$ transformed by $A_{\varphi}$.

The following theorem clearly implies Theorem 1:

THEOREM $1^{\prime}$. The motions $T_{\phi(x)} R_{\phi(x) ;(1)}$ with $0<x<1$ are free generators of a free group without fixed points.

Occasionally it is easy to derive the following corollary to this theorem.

COROLLARY. The rotations $R_{\phi(x) p(1)}$ with $0<x<1$ are free generators of a free group.

(This was proved by J. de Groot [5], Theorem II.)

4. For proving Theorem $I^{\prime}$ we need some lemmas.

LEMмA 1 . The values of the function $f(x)$ for $x>0$ are algebraically independent numbers, i. e., if we put different values of this function (which is strictly increasing) in the places of the arguments of a non-constant rational function with integral coeficients, then we obtain a transcendental number. 
This is a theorem of J. von Neumann [10].

LEMLA 2. Any product of the form

$$
A_{\varphi}^{k_{1}} B_{q}^{l_{1}} A_{\%}^{k_{3}} B_{q}^{L_{2}} \ldots A_{q}^{k_{n}} B_{\varphi}^{h_{n}}
$$

where $n \geqslant 1$ and $k_{i}$ and $l_{i}$ are integers different from 0 , except $k_{1}$ and $l_{n}$ one of which can be equal to 0 , is a non-constant function of $\varphi$.

This Lemma is proved in [5], p. 257-8.

We put for brevity

and suppose that

(4)

$$
\varphi=\varphi\left(x_{i}\right) \quad(i=1, \ldots, n)
$$

$$
x_{i} \neq x_{i+1} \quad \text { and } \quad 0<x_{i}<1 .
$$

LEMMA 3. Any product of the form

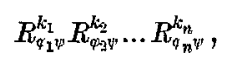

where $n \geqslant 1$ and $k_{i}$ are integers different from 0 is a non-constant function of $\psi$.

Proof. We write the product more explicitly

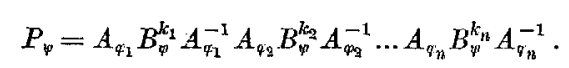

Of course

$$
P_{0}=I\left({ }^{2}\right)
$$

Then for proving the lemma it is enough to verify that

$$
P_{\varphi(x)} \neq 1 \quad \text { if } \quad x \neq x_{i} \quad \text { for } \quad i=1, \ldots, n \text {. }
$$

By (2) we have

$$
\sin \varphi(u)=\frac{2 f(u)}{1+(f(u))^{2}}, \quad \cos \varphi(u)=\frac{1-(f(u))^{2}}{1+(f(u))^{2}} .
$$

Therefore the elements of the matrix $P_{q(x)}$ are rational functions of the arguments $f\left(x_{1}\right), \ldots, f\left(x_{n}\right), f(x)$. By Lemma 1 it is enough to verify that one of them is a non-constant function, because then it gives a transcendental value. This is equivalent to the assertion that the product

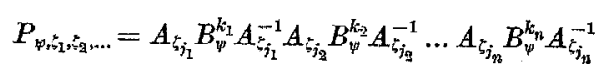

where $j_{r}=j_{s}$ if and only if $x_{r}=x_{s}$, is a non-constant function of the variables $\psi, \zeta_{1}, \zeta_{2}, \ldots$

(2) $I$ denotes the unity-matrix.
We have

$$
P_{q, q, 2, q, 3 \%, \ldots}=A_{q}^{i_{1}} B_{q}^{k_{1}} A_{q}^{j_{2}-j_{1}} B_{q}^{k_{2}} A_{q}^{j_{q}-j_{2}} \ldots A_{q}^{j_{n}-j_{n-1}} B_{q}^{k_{n}} A_{q}^{j_{n}}
$$

where by (4) $j_{r}-j_{r-1} \neq 0$. Then by Lemma $2 P_{q, \varphi, 2 \varphi, 3 p, \ldots}$ is a non-constant function of $\varphi$; which concludes the proof.

5. We introdnce the notations

$$
o=(0,0,0), \quad S_{\varphi \psi}=T_{\varphi} R_{\varphi \psi}, \quad p_{r q}=A_{\varphi}(1,0,0) .
$$

For any $p \in \mathcal{G}^{3}$ we denote by $[p]$ the translation defined by

Then $T_{q}=\left[p_{q}\right]$ and

$$
[p](q)=q+p\left(^{3}\right)
$$

$$
S_{q \psi}=\left[p_{\varphi}\right] R_{\tau \psi}
$$

$p_{q}$ is an eigenvector of $R_{F v}$

(becanse $(1,0,0)$ lies on the axis of the rotation $B_{y}$ ).

For any motion $M$ of $\mathcal{E}^{3}$ we consider the canonical decomposition

$$
M=[p] R
$$

where $p=M(0)$ and $R$ is a rotation around 0 .

(7) $M$ is without fixed points if and only if $p \neq 0$ and $p$ is not perpendicular to the axis of $R$ (if $R \neq e$ ).

We have the following rules for the multiplication of canonical decompositions:

(8) If $p$ is an eigenvector of $R$, then $([p] R)^{k}=[k p] R^{k}$ for $k=0, \pm 1, \pm 2, \ldots$

$$
\left[p_{1}\right] R_{1}\left[p_{2}\right] R_{2}=\left[p_{1}+R_{1}\left(p_{2}\right)\right] R_{1} R_{2} .
$$

LeMra 4. For any integers $k_{1}, \ldots, k_{n}$, we have the canonical decomposition.

$$
S_{q_{1} \eta}^{k_{1}} \ldots S_{q_{n} \psi}^{k_{\eta_{p}}}=\left[q_{\eta}\right] Q_{\eta}
$$

where

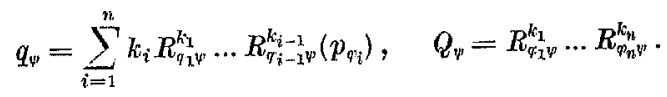

Proof. Of, course (5) is a canonical decomposition of $S_{q, p}$. Then applying (6), (8) and (9) we obtain the lemma.

6. Let $v_{v}$ denote the angular velocity vector of the rotation $Q_{v}$ at the moment $\psi(\psi$ is the time variable $)$. 
LEMva 5. $q_{*}=v_{\psi}$ and this vector either is a non-constant function of $\psi$ or is a constant $\neq 0$.

Proof. $v_{\psi}$ is the only vector which satisfies the equation

Now

$$
\frac{\partial}{\partial \psi} Q_{\psi}(p)=v_{\psi} \times Q_{\psi}(p)\left(^{4}\right) \quad \text { for each } \quad p \epsilon \mathcal{E}^{3} .
$$

and then

$$
\begin{aligned}
\frac{\partial}{\partial \psi} R_{q_{i} \psi}^{k_{i}}(p) & =\frac{\partial}{\partial \psi} A_{\psi_{i}} B_{\psi}^{k_{i}} A_{q_{i}}^{-1}(p)=A_{\varphi_{i}} \frac{\partial}{\partial \psi} B_{\psi}^{k_{i}}\left(A_{q_{i}}^{-1}(p)\right) \\
& =A_{q_{i}}\left(\left(k_{i}, 0,0\right) \times B_{\psi}^{k_{i}} A_{q_{i}}^{-1}(p)\right)=k_{i} p_{{q_{i}}_{i}} \times R_{\varphi_{i} \psi}^{k_{i}}(p)
\end{aligned}
$$

$$
\begin{aligned}
& \frac{\partial}{\partial \psi} Q_{\psi}(p)=\frac{\partial}{\partial \psi}\left(R_{\eta_{1} \psi}^{k_{1}} \ldots R_{q_{n} \psi}^{k_{n}}(p)\right)
\end{aligned}
$$

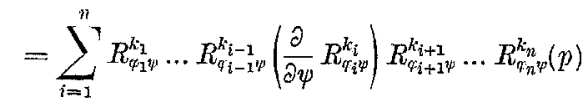

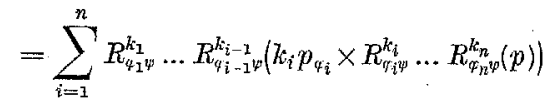

$$
\begin{aligned}
& =\sum_{i=1}^{n} k_{i} R_{\psi_{1} p}^{k_{1}} \ldots R_{q_{i-1}}^{k_{i-1}}\left(p_{q_{i}}\right) \times Q_{\psi}(p) \\
& =q_{p} \times Q_{\psi}(p) \text {. }
\end{aligned}
$$

Then the equality of Lemma 5 follows. By Lemma 3 it cannot be $v_{y} \equiv 0$, which concludes the proof.

7. Of course the elements of the matrix $Q_{\psi}$ are analytic functions of the variable $\psi$ and $Q_{0}=I$. Then by Lemma 3 there exists such an open non-empty interval $(0, \alpha)$ that

$$
Q_{y} \neq I \quad \text { for } \quad \psi \in(0, \alpha) \text {. }
$$

Let us denote by $l_{\varphi}$ any eigenvector $\neq o$ of the rotation $Q_{\varphi}$ (for $\psi \epsilon(0, \alpha)$ ).

LEMCA 6. There exists such a $\psi \in(0, \alpha)$ that $v_{\psi} \neq 0$ and $\Varangle\left(v_{\psi}, l_{\psi}\right) \neq \frac{1}{2} \pi$.

Proof. Since the coordinates of $v_{\psi}$ are analytic functions of $\psi$, by Lemma 5 there exists such an $\alpha^{\prime} \epsilon(0, \alpha)$, that

Suppose that

$$
v_{p} \neq 0 \text { for } \psi \in\left(0, \alpha^{\prime}\right) \text {. }
$$

$$
\Varangle\left(v_{\varphi}, l_{\psi}\right)=\frac{1}{2} \pi \quad \text { for each } \quad \psi \in\left(0, \alpha^{\prime}\right) .
$$

(4) $x$ denotes the vector product.
Then, since $l_{\psi}=Q_{\psi}\left(l_{\psi}\right)$,

$$
女\left(\left(v_{\psi}, Q_{\psi}\left(l_{\psi}\right)\right)=\frac{1}{2} \pi\right. \text {. }
$$

Now both derivatives $\partial v_{u} / \hat{\imath} u, \partial Q_{u}\left(l_{\psi}\right) / \partial u$ exist at the point $u=0$ (by analyticity of the functions). Then for every positive $\varepsilon$ there exists such a $\delta \in\left(0, \alpha^{\prime}\right)$ that if

then

$$
0<\chi<\psi<\delta
$$

$$
\Varangle\left(v_{\psi}, v_{x}\right)<\varepsilon \quad \text { and } \quad \Varangle\left(Q_{\psi}\left(l_{\psi}\right), Q_{x}\left(l_{\psi}\right)\right)<\varepsilon \text {. }
$$

Consequently, by (12), for every positive $\varepsilon$ there exists such a $\delta \in(0, \alpha)$ that if (13) holds then

That is

$$
\begin{gathered}
v_{\psi^{\prime}} \times Q_{\psi^{\prime}}\left(l_{\eta^{\prime}}\right) \neq 0, \quad v_{\chi} \times Q_{\chi}\left(l_{\psi^{\prime}}\right) \neq 0, \\
\Varangle\left(\left(v_{\psi^{\prime}} \times Q_{\psi^{\prime}}\left(l_{\psi^{\prime}}\right), v_{\chi} \times Q_{\chi}\left(l_{\psi}\right)\right)<\varepsilon .\right.
\end{gathered}
$$

$$
\begin{gathered}
{\left[\frac{\partial}{\partial u} Q_{u}\left(l_{\eta}\right)\right]_{u=\psi} \neq 0, \quad\left[\frac{\partial}{\partial u} Q_{u}\left(l_{\psi}\right)\right]_{u=\chi} \neq 0,} \\
\Varangle\left(\left[\frac{\partial}{\partial u} Q_{u}\left(l_{\psi}\right)\right]_{u=\psi},\left[\frac{\partial}{\partial u} Q_{u}\left(l_{\psi}\right)\right]_{u=\gamma}\right)<\varepsilon .
\end{gathered}
$$

This shows that for some $\varepsilon<\frac{1}{2} \pi$ and $0<\chi<\psi<\delta(\varepsilon)$ the projection of $\left[\partial Q_{u}\left(l_{\eta}\right) / \partial u\right]_{u=\chi}$ on $\left[\curvearrowright Q_{u}\left(l_{w}\right) / \partial u\right]_{u=\psi}$ is positive. This implies that

$$
Q_{0}\left(l_{\psi^{\prime}}\right) \neq Q_{\psi}\left(l_{\psi^{\prime}}\right) \quad \text { i. e. } \quad l_{\psi} \neq l_{\psi^{\prime}} .
$$

Then (11) is inconsistent, which proves the Lemma.

8. Of course $Q_{\psi}$ can be represented by an orthogonal matrix

$$
Q_{\psi}=\left(\alpha_{i j}\right)_{i, j=1,2,3} .
$$

Let $\left(\beta_{i j}\right)=\left(\alpha_{i j}\right)-I$ and let $\gamma_{i j}$ denote the algebraic complement of $\beta_{i j}$ in the matrix. $\left(\beta_{i j}\right)$.

It is clear that

(14) the vectors $l_{i}^{(i)}=\left(\gamma_{i 1}, \gamma_{i 2}, \gamma_{i 3}\right), i=1,2,3$, are eigenvectors of $Q_{*}$ and that if $Q_{\psi} \neq I$, at least one of them is different from 0 .

LEMMa 7. The sum

$$
\Sigma_{w}=\sum_{i=1}^{3}\left(v_{w} \cdot l_{\psi}^{(\eta)}\right)^{2}(5)
$$

either is a non-constant function of $\psi$ or is a constant $\neq 0$.

(5) - denotes the scalar product. 
Proof. Taking a number $\psi$ satisfying Lemma 6 we obviously have $\Sigma_{\psi} \neq 0$.

9. Proof of Theorem $1^{\prime}$. It is enough to show that every product of the form $(10)$, with $k_{i} \neq 0, n \geqslant 1$, and $\psi=\varphi(1)$ is a motion without fixed points. By Lemma 7 the expression $\Sigma_{\psi}$, which is a rational function of the arguments $f\left(x_{1}\right), \ldots, f\left(x_{n}\right)$ and $\operatorname{tg} \frac{1}{2} \psi$ (see (2), (3) and Lemma 5 ) is a non-constant function of $\psi$ or a constant $\neq 0$. Then in the first case by Lemma 1 and in the other case also,

$$
\Sigma_{q(1)} \neq 0
$$

(because $x_{i} \neq 1$ for $i=1, \ldots, n$ ). In the same way, by Lemmas 3 and 4 ,

$$
Q_{q(1)} \neq I \quad \text { and } \quad q_{q(1)} \neq 0 \text {. }
$$

By Lemma $5,(14)$ and (16) the vector $q_{\varphi(1)}$ is not orthogonal to the axis of the rotation $Q_{q(1)}$, which proves (see $(7)$ ) that the motion $\left[q_{\varphi(1)}\right] Q_{q(1)}$ is without fixed points; q. e. d.

10. Now we shall prove theorem 2 .

We adopt the notations introduced in section 1. Moreover:

Let $\left\{\varphi_{\mu}\right\}_{\mu \in M}\left(\varphi_{\mu_{1}} \neq \varphi_{\mu_{2}}\right.$ if $\left.\mu_{1} \neq \mu_{2}\right)$ be a set of free generators of a free group $\Phi$.

Then every $a \in \Phi$ has a unique factorization

$$
a=\varphi_{\mu_{\mathrm{I}}}^{k_{\mathrm{I}}} \ldots \varphi_{\mu_{n}}^{k_{n}}
$$

where $k_{i}= \pm 1$, and $\varphi_{\mu_{i}}^{k_{i}} \neq \varphi_{i_{i+1}}^{-k_{i+1}}$.

LEMMA. There exists a decomposition of $\Phi$ into $\overline{\bar{N}}$ disjoint sets $\left\{S_{y,\}_{w}, N}\right.$, one of which - say $A_{v_{0}}$ - is non-empty and satisfying the system of equalities

$$
\varphi_{\mu}\left(\bigcup_{\nu \in P_{\mu}} S_{\nu}\right)=\bigcup_{\nu \in Q_{\mu}} S_{\nu}, \quad \mu \in M .
$$

Proof. We take the notations

$$
P_{\mu}^{1}=P_{\mu}, \quad Q_{\mu}^{1}=Q_{\mu}, \quad P_{\mu}^{-1}=Q_{\mu}, \quad Q_{\mu}^{-1}=P_{\mu} .
$$

Then we must have

$$
p_{\mu}^{k}\left(\bigcup_{, \in P_{\mu k}^{p_{i}^{k}}} S_{\nu}\right)=\bigcup_{\nu \in Q_{\mu}^{k}} S_{\nu} \quad \text { for } \quad k= \pm 1
$$

We begin by putting $e$ into $S_{v_{0}}$. Now if $\alpha$ has been put into a set $S_{n_{0}}$ and $\beta=\varphi_{\mu}^{k} \alpha$ where $\varphi_{\mu}^{k}$ does not cancel with the first factor of $\alpha$, then we put $\beta$ into a set $S_{\nu_{2}}$ such that $\left(\nu_{1} \in P_{\mu}^{k} \& \nu_{2} \in Q_{u}^{k}\right)$ or $\left(\nu_{1} \in N: P_{k}^{k} \& \nu_{2} \in N \backslash Q_{\mu}^{k}\right)$.

Consequently (18) holds. Then the whole group $\Phi$ is decomposed $\left({ }^{6}\right)$, $S_{v_{0}} \neq \emptyset$ and $(17)$ is satisfied; q. e. d.

\footnotetext{
(") The axiom of choice is used here.
}

Proof of Theorem 2(7). Take the free group $\Phi$ without fixed points, with free generators $\left\{\varphi_{\mu}\right\}_{\mu \in M}$ given by Theorem 1 , and the factor space $\mathscr{E}^{3} / \Phi$. For any $E \in \mathcal{E}^{3} / \Phi$ we take a point $p_{E \in E}\left({ }^{6}\right)$.

Now using the Lemma we put

$$
A_{v}=\bigcup_{E} S_{v}\left(p_{E}\right)=\left\{p: p=\alpha\left(p_{E}\right), \alpha \in S_{v}, E \in \mathscr{E}^{3} / \Phi\right\} \text {. }
$$

Then, since $\Phi$ is without fixed points the pieces $A_{p}$ are disjoint and

$$
\varphi_{\mu}\left(\bigcup_{\nu \in P_{\mu}} A_{\nu}\right)=\bigcup_{E}\left(\varphi_{\mu} \bigcup_{\nu \in P_{\mu}} S_{\nu}\left(p_{E}\right)\right)=\bigcup_{E} \bigcup_{\nu \in Q_{\mu}} S_{\nu}\left(p_{E}\right)=\bigcup_{\nu \in Q_{\mu}} A_{\nu} .
$$

This proves the first part of the theorem.

It is easy to see that we can suppose that $\overline{\overline{\mathcal{E}^{3} / \Phi}}=2^{\mathrm{x}_{0}}$ (removing from $\Phi$ some of the generators). Then for different $E \in \mathcal{E}^{3} / \Phi$ we can take different $\nu_{0}$ in such a way that, if $\overline{\bar{N}} \leqslant 2^{\mathrm{x}_{0}}$, all the pieces $A_{v}$ are non-empty; which completes the proof of Theorem 2 .

\section{References}

[1] S. Balcerzyk and $\mathrm{Jan} \mathrm{MYciel}$ six, On the existence of free subgroups in topotogical groups, Fund. Math. 44 (1957), p. 303-308.

[2] Th. J. Dekker, Decompositions of sets and spaces I, II, Indagationes Math. $18(1956)$, p. 581-595.

[3] - Decompositions of sets and spaces III, Indagationes Math. 19 (1957), p. 104-107,

[4] - and $J$. de Groot, Decompositions of a sphere, Fund. Math. 43 (1955), p. 185-194.

[5] J. de Groot, Orlhogonal isomorphic representations of free groups, Canadian J. Math. 8 (1956), p. 256-262.

[6] Jan Mycielski, On the decompositions of Euclidean spaces, Bull. Acad. Polon. Sci., Cl. III, 4 (1956), p. 417-418.

[7] - About sets invariant with respect to denumerable changes, Fund. Math. this volume, p. 296-305.

[8] - On the paradox of the sphere, Fund. Math. 42 (1955), p. 348-355.

[9] - About sets with strange isometrical properties (I), Fund. Math. 42 (1955), p. 1-10.

[10] J. von Neumann, Ein System algebraisch unabhängiger Zahlen, Math. Ann. 99 (1928), p. 134-141.

[11] R. M. Robinson, On the decomposition of the spheres, Fund. Math. 42 (1947), p. 246-260.

[12] W. Sierpiński, Sur le paradoxe de la sphère, Fund. Math. 33 (1945), p. 235-244. Regu par la Rédaction le 18.7. 1957

(?) Compare [11] and [4]. 